\title{
Categorizing sounds by pitch: Effects of stimulus similarity and response repetition
}

\author{
TODD A. MONDOR, JENNIFER HURLBURT, and LESLIE THORNE \\ University of Manitoba, Winnipeg, Manitoba, Canada
}

\begin{abstract}
Two experiments were performed to determine whether categorization of the pitch of a probe tone is influenced by the pitch of, and response made to, a preceding prime tone. The prime and the probe could be drawn either from a pool of low-frequency sounds or from a pool of high-frequency sounds. The results of both experiments indicated that the performance obtained was best when the prime and the probe were the same pitch (and therefore required the same response), intermediate when the two sounds differed in pitch and required different responses, and slowest when the prime and the probe differed in pitch but required the same response (i.e., they were drawn from the same frequency pool). The results of Experiment 2 revealed in addition that when a repeated response was required, performance declined as the magnitude of the frequency change increased and that responses were made more quickly and accurately if the direction of the frequency change was away from the alternative category than if it was toward the alternative category. The results demonstrate that categorization of sounds by pitch is accomplished with reference to a previous processing episode.
\end{abstract}

A great many experiments have now been performed in which the ability of human listeners to differentiate sounds on the basis of pitch has been evaluated. Many studies have been directed toward determining the smallest frequency change that can be detected, and the evidence is clear that a change in frequency of as little as $2 \mathrm{~Hz}$ in a 1000-Hz standard tone may be detected reliably (e.g., Moore, 1989). In other studies, the way in which pitch perception may depend on the context in which a sound is presented has been examined. Such studies have revealed that a pure tone is masked most effectively by a sound of similar frequency (e.g., Moore, 1995; Zwislocki, 1978) and that the overall structure of a pattern may guide attention in such a way as to increase the likelihood that a pitch violation will be detected (e.g., Jones \& Yee, 1993). The influence of priming on pitch judgments has also been studied, with the evidence indicating that forewarning of location can facilitate judgments of the spectral composition of sounds (Mondor \& Zatorre, 1995) and that a suprathreshold pitch cue can substantially increase the probability of detecting a subsequent near-threshold pure tone of similar frequency (e.g., Creelman, 1959; Greenberg \& Larkin, 1968; Hübner \& Hafter, 1995; Larkin \& Greenberg, 1970; Scharf, Quigley, Aoki, Peachey, \& Reeves, 1987).

Thus, the available evidence suggests that pitch judgments may be influenced both by the context in which the

This research was supported by a grant from the Natural Sciences and Engineering Research Council of Canada to T.A.M. Correspondence may be addressed to T. A. Mondor, Department of Psychology, Duff Roblin Bldg., University of Manitoba, Winnipeg, MB, R3T 2N2 Canada (e-mail: todd_mondor@umanitoba.ca). judged sound is heard and by the pitch relation of a judged sound to one presented just prior to it. In contrast with this knowledge base, it appears that there has been no systematic investigation of the influence of an overt judgment of the pitch of one sound on the speed and accuracy of an overt judgment of the pitch of a subsequent sound. This is somewhat surprising given that extensive investigation of the influences of stimulus and response repetition on the time required to categorize visual stimuli has taken place. For example, Bertelson (1965) described an experiment in which four single digits were used as stimuli, with two of these ( 2 and 4$)$ assigned to one response and the other two (5 and 7) assigned to an alternative response. Bertelson was specifically interested in determining to what extent the time required to categorize a stimulus was influenced by the immediately preceding stimulus and the response made to it. This arrangement of stimuli and responses permitted Bertelson to study performance on three different types of trials: identical trials on which both the stimulus and the response were repeated, different trials on which both the stimulus and the response were changed, and equivalent trials on which the stimulus was changed but the response was repeated. Bertelson found that performance was slowest on different trials, with performance on identical trials slightly faster (by an average of about $20 \mathrm{msec}$ ) than on equivalent trials. These results clearly suggest that repeating a response improves performance substantially but that the additional benefit of repeating the stimulus as well is comparatively small.

Whereas Bertelson's (1965) study suggested that stimulus repetitions may be of relatively little importance in determining classification speed, subsequent experiments 
in which different stimuli have been used have provided results inconsistent with this conclusion. For example, M. C. Smith (1968) required her participants to categorize four visual stimuli, each consisting of a unique combination of the digits 1 and 2 and the colors red and green. Thus, one response was required for either a red 1 or a green 2, and a second response was required for either a green 1 or a red 2. In contrast with the results reported by Bertelson, M. C. Smith found that performance was best when both the stimulus and the response were repeated, intermediate when both the stimulus and the response were changed, and worst when the stimulus had been changed but a repeated response was required. M. C. Smith suggested that the results reported by Bertelson may have arisen because the two stimuli assigned to a response could be considered equivalent in their functional category, if not in their specific stimulus information. As M. C. Smith wrote, "it is possible that Ss coded the information in the form: even digit, left; odd digit, right, so that either a 2 or 4 activated the identical S-R code" (p. 436).

Although there is some inconsistency, then, in the results pertaining to the magnitude of the effects of stimulus and response repetition, there is clear agreement across studies that both of these factors influence categorization performance on serial, forced-choice tasks (see, also, Duncan, 1977; Fletcher \& Rabbitt, 1978; Hommell, 1998; Rabbitt, 1992; Rabbitt \& Vyas, 1979; Stoet \& Hommel, 1999; Walker \& Marshall, 1982). A variety of explanations have been offered both for the repetition effect (faster judgments of a repeated stimulus that requires a repeated response) and for the lengthening of response time (RT) when a repeated response is required to a different stimulus. One account that has been accepted by many theorists is that observers use a bypass rule in formulating a response to the second of two stimuli. The bypass rule is in essence a decision-making heuristic used to avoid a timeconsuming search of available stimulus-response (S-R) rules. Specifically, a check is made as to whether the stimulus is the same as the preceding stimulus. If so, the previous response may be repeated quickly. If not, the rule produces a tendency to execute a different response from that used on the previous trial. In describing the results reported by M. C. Smith (1968), Krueger and Shapiro (1981) argued that "the bypass rule could account for the sharp reduction she found in RT $(68 \mathrm{msec})$ on identical transitions, in which the subject would (correctly) regard the repetition of both stimulus aspects [i.e., colour and digit] as a cue to keep the same response, and for the sharp increase in RT ( $62 \mathrm{msec})$ on equivalent transitions, in which the subject would (incorrectly) regard the nonrepetition of both stimulus aspects as a cue to change the response" (p. 243; see also, e.g., Fletcher \& Rabbitt, 1978). In this way, the bypass rule may produce both quick responses to repeated stimuli and delayed responses to different stimuli that require a repeated response.

\section{The Present Study}

Irrespective of the specific theoretical explanation of these repetition effects, the results obtained have clearly established that the memory representation, or S-R link, generated when a visual stimulus is overtly judged may play an important role in subsequent judgments of visual objects. However, considerably less is known regarding the relation, if any, of one auditory judgment to another. The present study was designed with two goals in mind. First, in Experiment 1, we sought to determine whether both stimulus and response repetition would influence categorization of sounds on the basis of pitch. Second, in Experiment 2 , we wished to evaluate the possibility that when a repeated response is required, performance will be associated with the magnitude and direction of the frequency change. Answers to these questions would have clear implications for understanding the nature of the memory representation established when a response is executed to a sound and the influence of this representation on subsequent judgments.

\section{EXPERIMENT 1}

Experiment 1 was performed to determine whether stimulus and response repetition may influence the speed of pitch classification of pure tones. A consecutive response paradigm was used in which listeners were presented on each trial with two sounds in succession. They were required to make a pitch judgment for each of these sounds as quickly and accurately as possible. Four pure tones were used in the experiment, with two of these forming a low-pitched category $(500$ and $550 \mathrm{~Hz})$ and the other two forming a high-pitched category $(3000$ and $3300 \mathrm{~Hz})$. The inclusion of two sounds within each category permitted generation of three trial types defined by the pitch and response relation of the first sound (hereinafter, this sound will be referred to as the prime sound) to the second sound (hereinafter, this sound will be referred to as the probe sound). Following Bertelson (1965), we will refer to trials on which the prime and the probe sounds are the same as identical, trials on which the prime and the probe sounds differ in pitch but require the same response as equivalent, and trials on which the prime and the probe both differ in pitch and require different responses as different. Whereas a comparison of identical and different trials will permit an examination of the influence of stimulus-plus-response repetition, a comparison of identical and equivalent trials will provide an indication of the importance of pitch repetition over and above that of response repetition.

\section{Method}

\section{Participants}

Sixteen undergraduate students attending the University of Manitoba volunteered to participate in exchange for course credit. None of the participants reported any corrected or uncorrected hearing impairment.

\footnotetext{
Materials

Computer and sound system. The experiment was controlled by a Dell Pentium computer running the Maple programming environment (Bregman, Achim, \& Ahad, 1992). Sounds were presented in stereo over two Acoustic Profiles PSL 0.5 loudspeakers positioned $45^{\circ}$ to the left and right of the listener.
} 
Table 1

Response Times (RTs, in Milliseconds) and Percentages of Errors (\% E; With Standard Errors for Each Measure) as a Function of the Frequency and Response Relation of the Prime and the Probe Sounds in Experiment 1

\begin{tabular}{lccccc}
\hline & \multicolumn{2}{c}{$\mathrm{RT}$} & & \multicolumn{2}{c}{$\%$} \\
\cline { 2 - 3 } \cline { 5 - 6 } \multicolumn{1}{c}{ Trial Type } & $M$ & $S E$ & & $M$ & $S E$ \\
\hline Same frequency and same response & 346 & 13.61 & & 0.91 & 0.71 \\
Different frequency and same response & 404 & 22.92 & & 9.20 & 2.31 \\
Different frequency and different response & 374 & 18.30 & & 3.10 & 0.68 \\
\hline
\end{tabular}

Sounds. Four pure tones with frequencies of 500, 550, 3000, and $3300 \mathrm{~Hz}$, were used as stimuli in the experiment. All the sounds were synthesized using the MITSYN signal-processing software package (Henke, 1990) at $32000 \mathrm{~Hz}$. Each of the sounds was $50 \mathrm{msec}$ in duration and incorporated $3 \mathrm{msec}$ linear onset and offset amplitude ramps to eliminate onset and offset clicks. Sounds were presented to the listeners at a comfortable intensity of approximately $70 \mathrm{~dB}$ SPL as measured by a Brüel and Kjaer Model 2203 sound-level meter.

\section{Procedure}

The participants completed 24 practice trials and 192 experimental trials. On both the practice and the experimental trials, the participants were presented with two sounds in succession, and they were required to make a speeded frequency judgment (low or high) for each of these sounds. We will refer to the first of these sounds as the prime and to the second as the probe. The listeners made their responses by pressing either the "1" key on the keyboard to indicate that a sound was of low frequency or the " 0 " key to indicate that a sound was of high frequency. The participants were instructed to make their responses as quickly as possible while maintaining high accuracy. On practice trials, listeners were allowed 2,500 $\mathrm{msec}$ to respond, accuracy feedback was presented immediately after each response, and the listeners pressed the space bar to trigger presentation of each sound. In contrast, on experimental trials, the response window was only $750 \mathrm{msec}$, the time between the prime and the probe was set to 1,000 msec, no feedback was provided, and the listeners were required to initiate each trial by pressing the space bar after their response to the second sound on a trial.

There were three different types of trials, defined by the frequency and response relation of the prime and the probe. The prime and the probe were identical on one fourth of the trials (these will be referred to as identical trials), differed in frequency but required the same response on one fourth of the trials (these will be referred to as equivalent trials), and differed in frequency and required different responses on the remaining one half of the trials (these will be referred to as different trials). The specific frequencies chosen on each trial for the prime and for the probe were randomly selected, with the constraint that each of the four sounds was equally likely to be presented. Each of the 16 prime-probe combinations of the four frequencies was presented 12 times in the set of 192 trials. The 24 practice trials consisted of a random selection of the 16 unique frequency combinations, with the constraint that each of the four sounds was presented equally often in each of the prime and probe positions and that the prime and the probe were identical on $25 \%$ of the trials (identical), differed in frequency but required the same response on $25 \%$ of the trials (equivalent), and required different responses on $50 \%$ of the trials (different).

\section{Data Analysis}

Of primary interest was the influence of the frequency and response relation between the prime and the probe on frequency categorization of the probe. For this reason, performance on probe classification was examined only for trials on which a correct and acceptable response was made to the prime. Thus, trials on which the prime was misclassified or on which no response was made to the prime or the probe were discarded. Generally, performance in classifying the prime was quite accurate (mean accuracy of $89 \%$ in Experiment 1 and 92\% in Experiment 2), and there were few occasions on which no response was made to the prime or the probe (fewer than $2.5 \%$ in all the conditions in both experiments), so only a small percentage of trials were dropped outright. In addition, for each condition, outlying RTs to the probe, defined as those RTs more than 2.5 standard deviations above or below the mean, were excluded from all the analyses. In no condition in either experiment did outlier elimination lead to the exclusion of more than $1.5 \%$ of the responses.

\section{Results}

The results obtained in Experiment 1, summarized in Table 1, provided clear evidence that the classification of probe pitch was influenced by both the prime pitch and the response made to the prime. Specifically, performance in classifying the pitch of the probe was best on identical trials, intermediate on different trials, and worst on equivalent trials. This summary is supported by the results of separate one-way within-subjects analyses of variance (ANOVAs) used to evaluate performance as indicated by RT and by errors. Statistical analysis of the RT data revealed a robust main effect of prime-probe relation $[F(2,30)=$ $12.60, p<.001]$. Planned comparisons indicated that performance of all three trial types differed significantly from one another, with RT being fastest for trials on which the prime and the probe were identical, intermediate when the frequencies of the two sounds were different and they required different responses, and slowest when the sounds differed in frequency but required the same response $(p<$ .025 in all cases). Following Bertelson (1965) and others (e.g., E. E. Smith, Chase, \& Smith, 1973), the effect of stimulus repetition was estimated by the difference between performances on identical trials ( $346 \mathrm{msec})$ and equivalent trials (404 msec), and the effect of response repetition when there was a stimulus change was estimated by the difference between performances on different trials $(374 \mathrm{msec})$ and equivalent trials. Such an analysis indicates that both effects are quite robust, with stimulus repetitions leading to a reduction in RT of approximately $58 \mathrm{msec}$ (i.e., a facilitative effect) and with response repetitions when there was a stimulus change leading to an increase in RT of approximately $30 \mathrm{msec}$ (i.e., an inhibitory effect).

The pattern of performance indicated by the classification errors made by the listeners replicated the pattern apparent for RTs, with a significant effect of prime-probe relation $[F(2,30)=12.41, p<.001]$. Performance on the individual trial types was ordered in the same way as that indicated by the RT data, with significant differences in all comparisons ( $p<.02$ in all cases).

\section{Discussion}

The combined influence of both stimulus and response repetition replicates results that have been obtained in studies of visual perception (e.g., M. C. Smith, 1968). In this, the results are also consistent with an episodic retrieval mechanism in which the memory representation or 
event file incorporating information about both the preceding sound presented and the response executed to it exerts a significant influence on the speed with which subsequent judgments are made. One theoretical interpretation of this effect is in accordance with the bypass rule described earlier. According to this rule or heuristic, a new sound whose perceptual characteristics match the preceding sound may be responded to quickly because the bypass rule will engender a tendency to repeat the previous response. In contrast, when a new event that differs from the previous one in a basic perceptual feature, such as pitch, is presented, the bypass rule would engender a tendency to execute a different response.

\section{EXPERIMENT 2}

An open question, and one that appears not to have been addressed in studies of visual perception, is whether the degree to which RT is slowed on equivalent trials depends on the magnitude of the stimulus change. This is a theoretically important issue because, in the context of the present study, it speaks to the influence on performance of the specific pitch relation of the current and previous sounds. One possibility is that the bypass rule returns a repeat response tendency if there is no change in pitch and a change response tendency if there is a change in pitch. A fixed criterion, perhaps corresponding to the outer boundary of the critical band, might be used in determining whether or not there has a been a significant pitch change. Presumably, such a qualitative analysis of the pitch relation of successive sounds would be unaffected by the magnitude of the pitch change so long as the change was large enough to be detected. A second possibility is that the probability of the bypass rule's returning a same or a different response tendency may depend on the magnitude of the frequency change. According to this view, the likelihood of the bypass rule's returning a repeat response tendency will vary from nearly always for no pitch change at all to nearly never for a large pitch change. Thus, performance on equivalent trials should depend on the magnitude of the pitch difference between prime and probe sounds, with quite small pitch differences producing faster RTs than do larger changes, because of a higher probability that a repeat response outcome will arise from application of the bypass rule. Experiment 2 was designed to evaluate these possibilities. To this end, the design used in Experiment 1 was modified somewhat so that the magnitude of the pitch change was manipulated on equivalent trials. This design afforded opportunities both to replicate the results obtained in Experiment 1 and to determine whether performance on all equivalent trials is, indeed, equivalent.

\section{Method}

\section{Participants}

Twenty-four undergraduate students attending the University of Manitoba volunteered to participate in exchange for course credit. None of the participants reported any corrected or uncorrected hearing impairment.

\section{Materials}

Computer and sound system. The computer and sound system were the same as those used in Experiment 1.

Sounds. A low-pitched pool of seven pure tones (frequencies of $455,470,485,500,516,533$, and $550 \mathrm{~Hz}$ ) and a high-pitched pool of seven pure tones (frequencies of 2727, 2815, 2906, 3000, 3097, 3197 , and $3300 \mathrm{~Hz}$ ) were used as stimuli in this experiment. All the sounds were synthesized using the MITSYN signal-processing software package (Henke, 1990) at $32000 \mathrm{~Hz}$ and were $50 \mathrm{msec}$ in duration, including 3-msec linear onset and offset amplitude ramps.

\section{Procedure}

Experiment 2 was founded on the same three main trial types used in Experiment 1. In contrast with Experiment 1, however, the frequency of the prime was limited to be either 500 or $3000 \mathrm{~Hz}$, with each of these presented equally often. Each of the 14 total sounds could be presented as the probe, with the constraint that there were 48 identical trials on which the prime and the probe were the same pitch, 48 equivalent trials on which the probe was a different pitch from the prime but was drawn from the same frequency category, and 96 different trials on which the prime and the probe were drawn from different pitch categories. Thus, across trials, the probability of repeating a response was equal to the probability of changing the response. For equivalent trials, there were 16 trials at each of three frequency separations (small, medium, and large). On small-change trials, the $500-\mathrm{Hz}$ prime was followed by either a $485-$ or a $516-\mathrm{Hz}$ probe, and the $3000-\mathrm{Hz}$ prime was followed by either a $2906-$ or a $3097-\mathrm{Hz}$ probe. On medium-change trials, the $500-\mathrm{Hz}$ prime was followed by either a $470-$ or a $533-\mathrm{Hz}$ probe, and the $3000-\mathrm{Hz}$ prime was followed by either a $2815-$ or $3197-\mathrm{Hz}$ probe. On large-change trials, the $500-\mathrm{Hz}$ prime was followed by either a $455-$ or a $550-\mathrm{Hz}$ probe, and the $3000-\mathrm{Hz}$ prime was followed by either a $2727-$ or a $3300-\mathrm{Hz}$ probe. The magnitudes of the three sizes of frequency change were equivalent in log scale for the low-pitched and the high-pitched categories and for changes above or below the pitch of the prime. It is important to note that the smallest frequency change used in the experiment was substantially larger than the threshold for the minimum frequency change necessary for detection, which is less than $5 \mathrm{~Hz}$ for a $500-\mathrm{Hz}$ standard and $30 \mathrm{~Hz}$ for a $3000-\mathrm{Hz}$ standard (see, e.g., Shower \& Biddulph, 1931; Wier, Jesteadt, \& Green, 1977). However, even the largest frequency change on equivalent trials was no more than equal to the outer boundary of the critical band for the prime frequency (see, e.g., Zwicker, Flottorp, \& Stevens, 1957). The listeners completed 24 practice and 192 experimental trials. All other procedural details were the same as those in Experiment 1.

\section{Results}

The results obtained in Experiment 2 both replicated the results of Experiment 1 and demonstrated that the magnitude of the frequency change on equivalent trials is indeed an important determinant of performance. Specifically, as is shown in Tables 2 and 3, although average performance on equivalent trials was slower and less accu-

Table 2

Response Times (RTs, in Milliseconds) and Percentages of Errors (\% E; With Standard Errors for Each Measure) as a Function of the Frequency and Response Relation of the Prime and the Probe Sounds in Experiment 2

\begin{tabular}{lccccc}
\hline & \multicolumn{2}{c}{$\mathrm{RT}$} & & \multicolumn{2}{c}{$\%$} \\
\cline { 2 - 3 } \cline { 6 - 7 } \multicolumn{1}{c}{ Trial Type } & $M$ & $S E$ & & $M$ & $S E$ \\
\hline Same frequency and same response & 317 & 8.13 & & 1.05 & 0.31 \\
Different frequency and same response & 382 & 15.05 & & 6.83 & 0.94 \\
Different frequency and different response & 342 & 10.04 & & 3.83 & 0.88 \\
\hline
\end{tabular}


Table 3

Response Times (RTs, in Milliseconds) and Percentages of Errors (\% E; With Standard Errors for Each Measure) on Equivalent Trials as a Function of the Degree and Direction of Frequency Change Between the Prime and the Probe Sounds in Experiment 2

\begin{tabular}{|c|c|c|c|c|c|c|c|c|c|c|c|c|}
\hline \multirow[b]{4}{*}{ Direction } & \multicolumn{12}{|c|}{ Frequency Separation } \\
\hline & \multicolumn{4}{|c|}{ Small } & \multicolumn{4}{|c|}{ Medium } & \multicolumn{4}{|c|}{ Large } \\
\hline & \multicolumn{2}{|c|}{ RT } & \multicolumn{2}{|c|}{$\% \mathrm{E}$} & \multicolumn{2}{|c|}{ RT } & \multicolumn{2}{|c|}{$\% \mathrm{E}$} & \multicolumn{2}{|c|}{ RT } & \multicolumn{2}{|c|}{$\% \mathrm{E}$} \\
\hline & $M$ & $S E$ & $M$ & $S E$ & $M$ & $S E$ & $M$ & $S E$ & $M$ & $S E$ & $M$ & $S E$ \\
\hline Toward alternative category & 369 & 19.70 & 6.77 & 2.19 & 412 & 23.48 & 12.86 & 2.45 & 434 & 20.93 & 17.39 & 3.69 \\
\hline Away from alternative category & 339 & 13.50 & 0.52 & 0.52 & 358 & 12.51 & 1.22 & 0.85 & 376 & 13.86 & 2.23 & 1.04 \\
\hline
\end{tabular}

rate than performance on identical and different trials, this pattern depended on the magnitude of the frequency change on equivalent trials. When the frequency change was small, performances on equivalent trials and on different trials did not differ significantly. In contrast, both medium and large frequency changes slowed performance on equivalent trials considerably, so that performance on different trials was significantly faster.

A preliminary one-way within-subjects ANOVA (primeprobe relation with three levels [identical trials, average performance across all equivalent trials, and different trials]) was used to determine whether the pattern of results obtained in Experiment 1 had been replicated. This analysis of the RT data confirmed that this was the case; the main effect of prime-probe relation was significant $[F(2,46)=26.37, p<.001]$, with planned comparisons indicating that performance for all three trial types differed significantly from one another $(p<.01$ in all cases $)$. Probe pitch was categorized most quickly on the identical trials, most slowly on the equivalent trials, and with inter- mediate speed on the different trials. The effect of stimulus repetition, as estimated by the difference between performances on identical trials $(317 \mathrm{msec})$ and equivalent trials (382 msec), was facilitative, since RT was reduced by $65 \mathrm{msec}$. In contrast, the effect of response repetition when there was a stimulus change, as estimated by the difference between performances on different trials ( $342 \mathrm{msec})$ and equivalent trials, was inhibitory, since RT was increased by $40 \mathrm{msec}$ when a response was repeated to different sounds.

Of course, the main purpose of Experiment 2 was to determine whether the magnitude of the frequency change on equivalent trials was important in determining the speed of probe frequency classification. To evaluate this possibility, performances on the three frequency separations were compared using a one-way within-subjects ANOVA. This analysis confirmed a strong effect of the magnitude of frequency change $[F(2,46)=13.84, p<$ $.001]$, an influence that is represented visually in Figure 1. More detailed analyses indicated that RT was signifi-

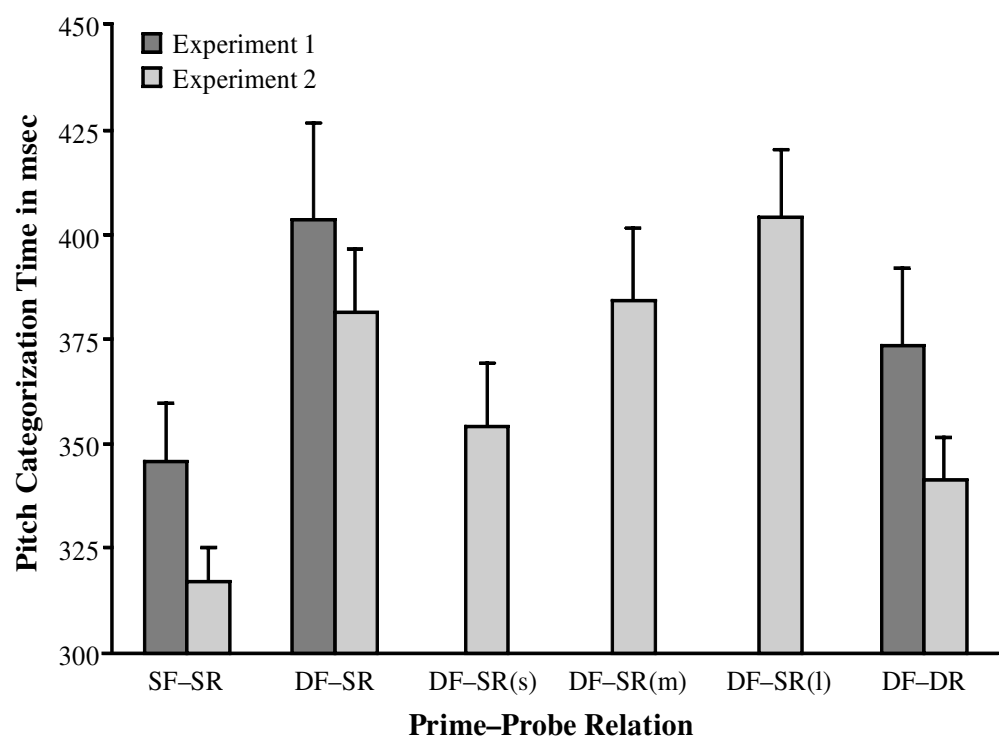

Figure 1. Pitch categorization time is represented as a function of the frequency and response relation between the prime and the probe sounds in Experiments 1 and 2. SF-SR, same frequency and same response; DF-SR, different frequency and same response; DF-SR (s), frequency difference and same response; DF-SR (m), medium frequency difference and same response; DF-SR (I), large frequency difference and same response; DF-DR, different frequency and different response. See the text for additional information. 
cantly slower on medium-change trials than on smallchange trials $(p<.01)$. Although RT was not significantly slower on large-change trials than on mediumchange trials $(p=.09)$, this null result appeared to have been due to a single participant who was $100 \mathrm{msec}$ faster on large-change trials than on medium-change trials. If the data from this listener are eliminated from the analysis, performance on large-change trials is significantly slower than that on medium-change trials $(p<.025) .{ }^{1}$ In any event, at minimum, the results establish that performance is better for small frequency changes than for medium or large changes.

A complementary analysis of the error data revealed the same results as those apparent for the RT data. Thus, there was a main effect of prime-probe relation $[F(2,46)=$ $17.32, p<.001]$, owing to significant differences in error rates between the three trial types ( $p<.015$ in all cases). The effect of the degree of stimulus change was also an important determinant of accuracy on equivalent trials $[F(2,46)=6.26, p<.01]$, with a significant increase in errors as the degree of pitch change increased from small to medium $(p<.05)$, but not from medium to large $(p=.15)$.

\section{Discussion}

These results establish that the magnitude of the frequency change significantly influences the speed and accuracy with which a pitch categorization judgment may be made. Specifically, when a repeated response is required, performance deteriorates as the size of the frequency change increases. This result is theoretically important in that it establishes that the process active in response determination is based on a quantitative, rather than a qualitative, consideration of frequency change. In particular, it is consistent with the possibility outlined above that the probability of the bypass rule's returning a repeat response tendency depends on the magnitude of the frequency change. According to this view, the faster performance on small pitch change trials than on medium and large pitch change trials results because the probability of the bypass rule's returning a repeat response tendency is higher. It appears clear that the bypass rule is not based on application of a fixed criterion, because if this were the case, there should not have been a linear change in RT as a function of frequency change on equivalent trials but, rather, a sharp change with equal and fast RT for pitch changes less than the criterion and equal and slower RTs for pitch changes greater than the criterion.

An alternative explanation of the effect of frequency change on equivalent trials is that the faster performance for small frequency transitions than for larger transitions may have arisen because the pitch change was detected less often. In other words, the listeners may have incorrectly perceived there to have been no frequency change on a larger proportion of small-transition than of larger transition trials. To evaluate this possibility, performance on equivalent trials was examined as a function of both the magnitude and the direction of the frequency change. We reasoned that if the effect of the magnitude of the fre- quency change apparent in our main analysis resulted because of differences in the probability that the listeners' misperceived the frequency change, the effect of frequency change should be the same whether the change is toward or away from the alternative category. To evaluate this possibility, a two-way within-subjects ANOVA (direction [toward other category or away from other category] $\times$ magnitude [small, medium, or large]) of RTs for equivalent trials was performed. This analysis revealed main effects of both direction $[F(1,23)=20.33, p<.001]$, and magnitude $[F(2,46)=13.84, p<.001]$, but no interaction between the two factors $[F(2,46)=1.16, p=.32]$. Overall, the listeners judged pitch more quickly and accurately when the pitch change was away from the alternative category. Separate consideration of performance for frequency changes toward and away from the alternate category showed strong effects of frequency change for both $[F(2,46)=8.08, p<.01$, and $F(2,46)=5.71, p<.01$, for shifts toward and away from the alternate category, respectively]. However, relative to performance on identical trials, there was a negative effect of frequency change irrespective of its direction ( $p<.001$ in both cases). A complementary analysis of the error data provided consistent results, with significant main effects of both magnitude $[F(2,46)=6.26, p<.001]$ and direction $[F(1,23)=24.40$, $p<.001]$, but with no interaction $[F(2,46)=2.61, p=$ .08]. In comparison with performance on different trials, the error rates on equivalent trials, in which the frequency shift was away from the alternate category, was significantly lower only when the change was small $(p<.01)$.

Clearly, as is shown in Table 3, the direction of the frequency change has a marked effect on performance on equivalent trials. Specifically, a frequency transition away from the alternative category leads to a substantially faster and more accurate response than does a frequency transition toward the alternative category. This in turn suggests that the influence of the magnitude of the stimulus change is not mediated by failures to perceive a physical change in pitch but, rather, arises because of specific consideration of both the magnitude and the direction of the pitch change. One might reasonably expect that such detailed analysis would yield particularly fast and accurate categorization responses on equivalent trials when the frequency change was away from the alternative category. However, relative to performance on different trials, on equivalent trials there was no beneficial effect of this detailed analysis apparent in RTs and a significant benefit in accuracy only when the frequency change was small $(p<.01)$, but not when it was medium $(p=.07)$ or large $(p=.30)$.

\section{GENERAL DISCUSSION}

The influence of attention on auditory processing (e.g., Cherry, 1953; Mondor, Breau, \& Milliken, 1998; Moray, $1959,1975)$ and the effect of spatial compatibility of sound location and the overt response (e.g., Simon, 1990; Simon, Craft, \& Small, 1971; Simon \& Rudell, 1967) have been quite well documented. However, remarkably little empir- 
ical effort has been directed toward delineating the influence of identifying and responding to one sound on identifying and responding to a subsequent sound. It is this specific influence that was of primary concern to us in the present paper. Taken together, the results of the two experiments provide clear evidence that pitch categorization performance is influenced both by the pitch of a preceding sound and by the response made to it. This effect was quite reliable in that, in both experiments, categorization was fastest for repeated sounds (identical trials), intermediate for sounds that differed in pitch from the preceding sound and required a different response (different trials), and slowest for sounds that differed in pitch from the preceding sound but required the same response (equivalent trials). Thus, average performance across the two experiments indicated that whereas there was a strong facilitative effect of stimulus repetition alone of about $62 \mathrm{msec}$ (as estimated by the difference in performance on identical and equivalent trials), there was a reliable inhibitory effect of response repetition when there was a pitch change of about $35 \mathrm{msec}$ (as estimated by the difference in performance on equivalent and different trials). Furthermore, the results of Experiment 2 revealed that both the magnitude and the direction of the pitch change on equivalent trials had a significant influence on performance, with listeners making their pitch classification judgments substantially more quickly and accurately for small pitch changes and when the direction of a pitch change was away from the alternative category. These results provided strong evidence that a detailed evaluation of the pitch relation of the prime and the probe sounds is executed as part of the response determination process. Such an influence of a prior processing episode provides indirect evidence that the memory representation or event file established when an explicit response is executed to a sound includes information both about the perceptual characteristics of the sound and about the overt response.

As we noted above, several investigators (e.g., Fletcher \& Rabbitt, 1978; Krueger \& Shapiro, 1981) have suggested that in a two-choice serial RT task such as that used in the two experiments described above, participants have a tendency to repeat a response to a repeated stimulus and to change their response when the stimulus changes. This heuristic, usually referred to as the bypass rule, has been offered as a potential explanation for the effects of both stimulus repetition and response repetition. The present results are certainly consistent with this viewpoint. It is interesting to note that evidence of the operation of this heuristic was obtained even though the stimuli varied only along the single dimension of pitch. This stands in sharp contrast with studies of stimulus and response repetition on the time required to classify visual objects, in which stimuli have typically varied on two or more dimensions (e.g., Bertelsen, 1965; Rabbitt, 1992). The possibility that our results indicate a special role of pitch information in overtly responding to a sound is a question we are now in the process of examining.

Clearly, in generating a response tendency in favor of either repeating a previous response or making a new re- sponse, application of a heuristic such as the bypass rule can significantly influence performance on a pitch classification task. It is equally clear, however, that final response determination is not governed only by this tendency; if it were, error rates would have been near $100 \%$ on equivalent trials, where the bypass rule would have produced an incorrect tendency to change the response. It appears clear, then, that application of a bypass rule produces a response tendency that is subject to a later error-checking process. Although an empirical consideration of such an evaluative process is beyond the scope of the present paper, as a working hypothesis we might assume that the response tendency produced by the bypass rule and the stimulus and response information available from examination of the previous processing episode would be used to determine the candidate $S-R$ mapping rule for initial evaluation. For example, on identical trials, the bypass rule would produce a repeat response $X$ suggestion, the evaluation process would confirm that executing response $X$ to stimulus $\mathrm{Y}$ is consistent with the mapping rules, and a quick response would be executed. In contrast, on different trials, the bypass rule would return a change response tendency, the evaluation process would need first to search for the available alternative responses, and only then would a specific S-R mapping rule be evaluated and affirmed. On this view, the additional requirement of searching for the alternative response would account for the modest increase in RT on different trials relative to identical trials. Finally, on equivalent trials, the bypass rule would produce a change response tendency, the evaluation process would search for the alternative response, and then the candidate $S-R$ rule would be evaluated and, in this case, rejected. At this point, the alternative mapping (i.e., repeat the previous response even though the stimulus has changed) would be evaluated and verified. Thus, the difference in RT on identical, different, and equivalent trials could be attributed to the joint influences of the bypass rule and the more specific evaluation of candidate S-R mappings. Although this primitive "model" is clearly speculative, it is consistent with the results reported above, and we hope that it may direct future research in productive directions.

In conducting this study, we set out to determine whether stimulus and response repetition influences categorization of sounds by pitch and, if so, whether performance depends on the degree to which pitch changes from one sound to another. The two experiments described above provide clear affirmative answers to both of these questions and, in this, suggest that application of the bypass rule entails a detailed consideration of stimulus change. At a more general level, the results indicate that even judgments of a fundamental auditory perceptual feature, such as pitch, are accomplished with reference to a prior processing episode.

\section{REFERENCES}

Bertelson, P. (1965). Serial choice-reaction time as a function to response versus signal-and-response repetition. Nature, 206, 217-218. Bregman, A. S., Achim, A., \& Ahad, P. A. (1992). The Maple software system. Montreal: McGill University.

Cherry, E. C. (1953). Some experiments on the recognition of speech, with one and with two ears. Journal of the Acoustical Society of America, 25, 975-979. 
Creelman, C. D. (1959). Detection of signals of uncertain frequency. Journal of the Acoustical Society of America, 32, 805-810.

Duncan, J. (1977). Response selection errors in spatial choice reaction tasks. Quarterly Journal of Experimental Psychology, 29, 415-423.

Fletcher, B. C., \& RabbitT, P. M. A. (1978). The changing pattern of perceptual analytic strategies and response selection with practice in a two-choice reaction time task. Quarterly Journal of Experimental Psychology, 30, 417-427.

Greenberg, G. Z, \& LARKIN, W. D. (1968). Frequency-response characteristic of auditory observers detecting signals of a single frequency in noise: The probe-signal method. Journal of the Acoustical Society of America, 44, 1513-1523.

HENKE, W. L. (1990). An interactive dialoguelanguagefor time signal processing. Cambridge, MA: MIT Research Laboratory of Electronics.

Hommell, B. (1998). Event files: Evidence for automatic integration of stimulus-response episodes. Visual Cognition, 5, 183-216.

HÜBNER, R., \& HAFTER, E. R. (1995). Cuing mechanisms in auditory signal detection. Perception \& Psychophysics, 57, 197-202.

Jones, M. R., \& YeE, W. (1993). Attending to auditory events: The role of temporal organization. In S. McAdams \& E. Bigand (Eds.), Thinking in sound: The cognitive psychology of human audition (pp. 69-112). Oxford: Oxford University Press, Clarendon Press.

Krueger, L. E., \& Shapiro, R. G. (1981). Intertrial effects of samedifferent judgements. Quarterly Journal of Experimental Psychology, 33A, 241-265.

LARKIN, W., \& GREenberG, G. Z. (1970). Selective attention in uncertain frequency detection. Perception \& Psychophysics, 8, 179-184.

Mondor, T. A., Breau, L. M., \& Milliken, B. (1998). Inhibitory processes in auditory selective attention: Evidence of location-based and frequency-based inhibition of return. Perception \& Psychophysics, 60, 296-302.

Mondor, T. A., \& Zatorre, R. J. (1995). Shifting and focusing auditory spatial attention. Journal of Experimental Psychology: Human Perception \& Performance, 21, 387-409.

Moore, B. C. J. (1989). An introduction to the psychology of hearing (3rd ed.). Toronto: Academic Press.

Moore, B. C. J. (1995). Frequency analysis and masking. In B. C. J. Moore (Ed.), Hearing: Handbookof perception and cognition (2nd ed., pp. 161205). San Diego: Academic Press.

Moray, N. (1959). Attention in dichotic listening: Affective cues and the influence of instructions. Quarterly Journal of Experimental Psychology, 12, 214-220.

Moray, N. (1975). A data base for theories of selective listening. In P. M. A. Rabbitt \& S. Dornic (Eds.), Attention and performance $V$ (pp. 119-135). New York: Academic Press.

RabBITT, P. M. A. (1992). Many happy repetitions: A celebration of the "Bertelson Repetition Effect" 1961-1991. In J. Alegria, D. Holender, J. Junca de Morais, \& M. Radeau (Eds.), Analytic approaches to human cognition (pp. 313-330). Amsterdam: North-Holland.
Rabbitt, P. M. A., \& Vyas, S. M. (1979). Memory and data-driven control of selective attention in continuous tasks. Canadian Journal of Psychology, 33, 71-87.

Scharf, B., Quigley, S., Aoki, C., Peachey, N., \& Reeves, A. (1987). Focused auditory attention and frequency sensitivity. Perception \& Psychophysics, 42, 215-223.

SHOWER, E. G., \& BIDDULPH, R. (1931). Differential pitch sensitivity of the ear. Journal of the Acoustical Society of America, 3, 275-287.

Simon, J. R. (1990). The effects of an irrelevant directional cue on human information processing. In R. W. Proctor \& T. G. Reeve (Eds.), Stimulus-response compatibility: An integrated perspective (Advances in Psychology, Vol. 65, pp. 31-86). Amsterdam: North-Holland.

Simon, J. R., Craft, J. L., \& Small, A. M. (1971). Reactions toward the apparent source of an auditory stimulus. Journal of Experimental Psychology, 89, 203-206.

Simon, J. R., \& Rudell, A. P. (1967). Auditory S-R compatibility: The effect of an irrelevant cue on information processing. Journal of Applied Psychology, 51, 300-304.

Smith, E. E., Chase, W. G., \& Smith, P. G. (1973). Stimulus and response repetition effects in retrieval from short-term memory: Trace decay and memory search. Journal of Experimental Psychology, 98, 413-422.

Sмiтн, M. C. (1968). Repetition effect and short-term memory. Journal of Experimental Psychology, 77, 435-439.

Stoet, G., \& Hommel, B. (1999). Action planning and the temporal binding of response codes. Journal of Experimental Psychology: Human Perception \& Performance, 25, 1625-1640.

WALKer, P., \& MARShall, E (1982). Visual memory and stimulus repetition effects. Journal of Experimental Psychology: General, 111, 348-368.

Wier, C. C., Jesteadt, W., \& Green, D. M. (1977). Frequency discrimination as a function of frequency and sensation level. Journal of the Acoustical Society of America, 61, 178-184.

Zwicker, E., Flottorp, G., \& Stevens, S. S. (1957). Critical bandwidth in loudness summation. Journal of the Acoustical Society of America, 29, 548-557.

ZWISLOCKI, J. J. (1978). Experimental and theoretical aspects of simultaneous, forward, backward, and central masking. In E. C. Carterette \& M. P. Freidman (Eds.), Handbook of perception: Vol. IV. Hearing (pp. 283-336). New York: Academic Press.

\section{NOTE}

1. As a check to determine whether the other significant results of Experiment 2 were an artifact of the performance of this 1 listener, all of the analyses reported in the Results section of Experiment 2 were repeated with the data from this participant excluded. None of the results changed.

(Manuscript received December 13, 2001; revision accepted for publication June 20, 2002.) 